\title{
Non-Obstetric Surgical Care at Three Rural District Hospitals in Rwanda: More Human Capacity and Surgical Equipment May Increase Operative Care
}

\author{
Ernest Muhirwa $^{1}$ - Caste Habiyakare ${ }^{2}$ Bethany L. Hedt-Gauthier ${ }^{1,3}$. \\ Jackline Odhiambo $^{1}$ - Rebecca Maine ${ }^{3,4}$ - Neil Gupta ${ }^{1,5}$ - Gabriel Toma ${ }^{1,3}$.

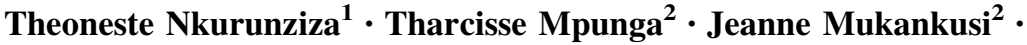 \\ Robert Riviello ${ }^{3,5,6}$
}

Published online: 20 April 2016

(C) Société Internationale de Chirurgie 2016

\begin{abstract}
Background Most mortality attributable to surgical emergencies occurs in low- and middle-income countries. District hospitals, which serve as the first-level surgical facility in rural sub-Saharan Africa, are often challenged with limited surgical capacity. This study describes the presentation, management, and outcomes of non-obstetric surgical patients at district hospitals in Rwanda.

Methods This study included patients seeking non-obstetric surgical care at three district hospitals in rural Rwanda in 2013. Demographics, surgical conditions, patient care, and outcomes are described; operative and non-operative management were stratified by hospitals and differences assessed using Fisher's exact test.

Results Of the 2660 patients who sought surgical care at the three hospitals, most were males $(60.7 \%)$. Many $(42.6 \%)$ were injured and $34.7 \%$ of injuries were through road traffic crashes. Of presenting patients, $25.3 \%$ had an operation, with patients presenting to Butaro District Hospital significantly more likely to receive surgery (57.0\%, $p<0.001)$. General practitioners performed nearly all operations at Kirehe and Rwinkwavu District Hospitals $(98.0$ and $100.0 \%$, respectively), but surgeons performed $90.6 \%$ of the operations at Butaro District Hospital. For outcomes, $39.5 \%$ of all patients were discharged without an operation, $21.1 \%$ received surgery and were discharged, and $21.1 \%$ were referred to tertiary facilities for surgical care.

Conclusion Significantly more patients in Butaro, the only site with a surgeon on staff and stronger surgical infrastructure, received surgery. Availing more surgeons who can address the most common surgical needs and improving supplies and equipment may improve outcomes at other districts. Surgical task sharing is recommended as a temporary solution.
\end{abstract}

Ernest Muhirwa and Caste Habiyakare are joint first authors.

Ernest Muhirwa

ernestinoy@gmail.com

Partners In Health/Inshuti Mu Buzima, Kigali, Rwanda

Ministry of Health, Kigali, Rwanda

3 Department of Global Health and Social Medicine, Harvard Medical School, Boston, MA, USA

4 Department of Surgery, University of California, San Francisco, CA, USA

5 Division of Global Health Equity, Brigham and Women's Hospital, Boston, MA, USA

6 University Teaching Hospital, Kigali, Rwanda

\section{Introduction}

About a quarter of the global burden of diseases can be treated with surgery [1] with injuries (38\%), malignancies (19\%), and congenital abnormalities $(9 \%)$ as the leading conditions needing surgical care [2]. Only a third of the world's population, mostly from high-income countries, benefit from three quarters of the 234.2 million major surgical procedures performed worldwide [2]. Approximately three quarters of mortality attributable to surgical emergencies are in low- and middle-income countries (LMICs) [3].

Although conditions needing minor and major surgery are common in LMICs [2, 4], the lack of access to surgical care remains a critical gap in these health systems [5]. In 
the rural areas of most LMICs, including Rwanda, district hospitals serve as the first-level facility providing surgical care [6]. However, inadequate expertise, equipment, and facilities in the district hospitals limit access, safe, and efficient surgical care $[2,6]$. Further, financial barriers such as related opportunity costs and cultural barriers such as fear of experiencing surgery and fear of negative outcomes from anesthesia inhibit patients from seeking care [5].

The nature of surgical procedures performed at district hospitals also vary. In 2009 and 2010, over 80,000 surgical procedures (major and minor) were recorded annually in Rwanda. Surgical registers and annual hospital reports at two referral hospitals in Rwanda showed that 82.5 per cent of major surgical procedures were performed in district hospitals [7]. However, $60 \%$ of these major operations were obstetrics and gynecologic. Little is known about the types and management of non-obstetric surgical conditions in the rural district hospitals. This study describes the management and outcomes of patients needing non-obstetric surgical care at three rural district hospitals in Rwanda. The understanding of the non-obstetrics surgical needs and care provided can stimulate changes in policy and practice guidelines that may improve surgical care and hence patient outcomes.

\section{Materials and methods}

\section{Study design and setting}

This retrospective cohort study included patients seeking non-obstetric surgical care from January 1 to December 31, 2013 at three rural district hospitals (Kirehe, Butaro and Rwinkwavu District Hospitals (DHs)) in Eastern and Northern Provinces of Rwanda. These hospitals are managed by the Ministry of Health and supported by Partners In Health/Inshuti Mu Buzima (PIH/IMB), a USbased non-governmental organization. Collectively, these hospitals serve a population of 897,117 people and provide surgical care to patients referred from 41 health centers.

In Rwanda, district hospitals provide basic surgical care, primarily minor and obstetric surgeries. These hospitals rely on general practitioners and nurses to provide surgical care, except for Butaro DH which had a general surgeon on staff for most of 2013 and benefited from frequent short-term visiting specialists such as plastic surgeons. In addition, Butaro is a cancer center of excellence, thus more equipped to provide surgical care. Typically, patients who need major surgical interventions at any of the three hospitals are referred to tertiary hospitals.

\section{Study population and data collection}

The study included all patients admitted to the DHs for nonobstetric surgical conditions from January 1 to December 31, 2013. Patients who were admitted for obstetric conditions were excluded. We collected data on demographics and surgical conditions at presentation, the operative and nonoperative management provided, and patient outcomes. We defined non-operative management as treatment, such as laboratory tests, imaging, narcotic and non-narcotic pain medication, wound care, and the use of antibiotics, that did not involve surgical operation. Data were extracted from patients' charts and from registers in surgery and theater departments. Four data collectors were trained in chart extraction and data entry. A sample of data was reviewed and matched across the admission information, patient charts, and theater logs for completeness and accuracy.

\section{Analysis and statistics}

We described the number and percent for demographic variables and surgical conditions. The operative and nonoperative management provided was stratified by hospitals, and differences were assessed using Fisher's exact test. We described overall patient outcomes, categorized as patients who were discharged after operative care, non-operative management, referred for tertiary care, absconded, or died. Stata v13 (College Station, TX: StataCorp LP) was used for analysis.

\section{Ethics}

The study received technical approval from the PIH/IMB Research Committee and the National Health Research Committee, and received ethical approval from Rwanda National Ethics Committee and the Partner's Human Research Committee in Boston, MA. The study was also approved by the Rwanda Ministry of Health.

\section{Results}

\section{Demographics}

The 2660 patients needing non-obstetric surgical care were relatively evenly distributed across the hospitals, with 766 (28.8\%) at Butaro DH, 909 (34.2 \%) at Rwinkwavu DH and $985(37.0 \%)$ at Kirehe DH (Table 1). For the 2647 (95.5\%) with gender recorded, most were males $(n=1607,60.7 \%)$, and for the 2573 (96.7 \%) with age recorded, 864 (33.6\%) were between 25 and 49 years. Insurance status was recorded for $1611(60.5 \%)$ of the patients, of which 1382 
Table 1 Characteristics and surgical conditions of patients seeking non-obstetric surgical care

\begin{tabular}{|c|c|c|}
\hline & $n$ & $\%$ \\
\hline Total number of patients & 2660 & 100.0 \\
\hline \multicolumn{3}{|l|}{ Hospital } \\
\hline Butaro & 766 & 28.8 \\
\hline Rwinkwavu & 909 & 34.2 \\
\hline Kirehe & 985 & 37.0 \\
\hline Gender & \multicolumn{2}{|c|}{$N=2647(95.5 \%)$} \\
\hline Male & 1607 & 60.7 \\
\hline Female & 1040 & 39.3 \\
\hline Age (years) & \multicolumn{2}{|c|}{$N=2573(96.7 \%)$} \\
\hline$\leq 15$ years & 748 & 29.1 \\
\hline$>15$ and $\leq 24$ years & 383 & 14.9 \\
\hline$>24$ and $\leq 49$ years & 864 & 33.6 \\
\hline$>49$ years & 578 & 22.5 \\
\hline Health insurance & \multicolumn{2}{|c|}{$N=1611(60.5 \%)$} \\
\hline Uninsured & 131 & 8.1 \\
\hline National insurance & 1382 & 85.8 \\
\hline Other & 98 & 6.1 \\
\hline \multicolumn{3}{|l|}{ Occupation } \\
\hline Farmer & 788 & 29.6 \\
\hline Business/employed & 54 & 2.0 \\
\hline Student/child & 684 & 25.7 \\
\hline Other & 85 & 3.2 \\
\hline Unknown & 1049 & 39.4 \\
\hline \multicolumn{3}{|l|}{ Home district } \\
\hline Within hospital catchment areas & 2229 & 83.8 \\
\hline Outside of hospital catchment areas & 431 & 16.2 \\
\hline Type of diagnosis & \multicolumn{2}{|c|}{$N=2405(90.4)$} \\
\hline Trauma & 1024 & 42.6 \\
\hline Infection & 540 & 22.5 \\
\hline Surgically resectable cancers & 252 & 10.5 \\
\hline General surgery $^{\mathrm{a}}$ & 508 & 21.1 \\
\hline Congenital defect & 32 & 1.3 \\
\hline Urology & 49 & 2.0 \\
\hline Types of trauma & \multicolumn{2}{|c|}{$N=939(91.7 \%)$} \\
\hline Road traffic injuries & 355 & 37.8 \\
\hline Fall & 202 & 21.5 \\
\hline Assault (blunt force/fight/stab/cut) & 219 & 23.3 \\
\hline Burns & 65 & 6.9 \\
\hline Other & 98 & 10.4 \\
\hline
\end{tabular}

${ }^{a}$ We define general surgery as a surgical specialty that focuses on the abdominal area including esophagus, stomach, small bowel, colon, liver, pancreas, gallbladder and bile ducts, and the thyroid gland (depending on local reference patterns). It also deals with diseases involving the skin, breast, soft tissue, and hernias. These are cases not already included in trauma, infection, or surgically resectable cancers

$(85.8 \%)$ had national insurance and $131(8.1 \%)$ were not insured. For the $2405(90.4 \%)$ with a final diagnosis, the largest cohort $(n=1024,42.6 \%)$ was trauma patients. For the 939 (91.7\%) of trauma patients with details on mechanism of injury recorded, a third $(n=355,37.8 \%)$ were caused by road traffic injuries.

\section{Operative care}

For the 2576 (96.8\%) with information recorded, 649 (25.3\%) had a surgical procedure completed at the district hospital, with patients at Butaro DH significantly more likely to have surgery $(n=436$ out of $765,57.0 \%$, $p<0.001$ ) (Table 2). Of the operations performed, 36 $(6.7 \%)$ were emergency surgeries with the remaining elective, with the percent of emergency operations in Rwinkwavu DH being significantly higher (17.4\%, $p=0.019)$. Most operations $(n=373,81.6 \%)$ were on patients in ASA Class I.

The most common operative procedures were excision of cysts, lipomas, keloids, and masses $(n=152,34.9 \%)$ at Butaro DH; abscess incision and drainage $(n=22$, $24.2 \%)$ at Rwinkwavu $\mathrm{DH}$; and hernia/hydrocele repair $(n=76,62.3 \%)$ at Kirehe DH. Most patients received antibiotics post-operatively in Rwinkwavu $(n=63$, $90.0 \%)$ and Kirehe $(n=67,77.9 \%)$ and peri-operatively in Butaro $(n=267,79.2 \%, p<0.001)$. The use of general anesthesia was more common at Rwinkwavu DH $(n=52,74.3 \%)$, followed by Kirehe DH $(n=52$, $53.6 \%)$. However, local anesthesia was more often used at Butaro DH $(n=111,26.6 \%)$ compared to the other sites.

The majority of procedures were performed by general practitioners at Kirehe $(n=118,100 \%)$ and Rwinkwavu DHs $(n=81,98.8 \%)$, where provider type was recorded. In contrast, at Butaro DH, 184 (42.6\%) of the surgical procedures were performed by the on-staff surgeon and 182 $(42.1 \%)$ were performed by visiting plastic surgeons. Most surgical procedures with duration reported were completed in less than thirty minutes $(n=143,47.4 \% ; n=15$, $51.7 \%$; and $n=46,69.7 \%$ for Butaro, Rwinkwavu and Kirehe DHs, respectively). Surgical complications were rarely recorded in over $85 \%$ of cases at each site.

\section{Non-operative management-evaluation and treatment}

For the 2660 patients, $1583(59.5 \%)$ had at least one laboratory test recorded and $1197(45.0 \%)$ patients had at least one imaging completed (Table 3). The use of laboratory evaluation differed between sites $(p<0.001)$. Butaro DH patients were most likely to have at least one laboratory test $(n=649,84.7 \%)$. The most common laboratory tests were full blood count $(n=1447,54.4 \%)$ and HIV tests $(n=913,34.3 \%)$. Imaging also differed significantly among the hospitals $(p<0.001)$ with Kirehe $\mathrm{DH}$ patients most likely to have at least one imaging 
Table 2 Operative interventions for patients needing non-obstetric surgical care

\begin{tabular}{|c|c|c|c|c|c|c|c|c|c|}
\hline & \multicolumn{2}{|c|}{ All the hospitals } & \multicolumn{2}{|c|}{ Butaro } & \multicolumn{2}{|c|}{ Rwinkwavu } & \multicolumn{2}{|c|}{ Kirehe } & \multirow[t]{2}{*}{$p$ value } \\
\hline & $n$ & $\%$ & $n$ & $\%$ & $n$ & $\%$ & $n$ & $\%$ & \\
\hline Operation performed & \multicolumn{2}{|c|}{$N=2576$} & \multicolumn{2}{|c|}{$N=765$} & \multicolumn{2}{|c|}{$N=871$} & \multicolumn{2}{|c|}{$N=931$} & \\
\hline Yes & 649 & 25.3 & 436 & 57.0 & 91 & 10.5 & 122 & 13.1 & $<0.001$ \\
\hline No & 1918 & 74.7 & 329 & 43.0 & 780 & 89.5 & 809 & 86.9 & \\
\hline \multicolumn{10}{|l|}{ For patients who received a surgical procedure } \\
\hline Operation urgency & \multicolumn{2}{|c|}{$N=537$} & \multicolumn{2}{|c|}{$N=401$} & \multicolumn{2}{|c|}{$N=46$} & \multicolumn{2}{|c|}{$N=90$} & \\
\hline Elective & 501 & 93.3 & 377 & 94.0 & 38 & 82.6 & 86 & 95.6 & 0.019 \\
\hline Emergency & 36 & 6.7 & 24 & 6.0 & 8 & 17.4 & 4 & 4.4 & \\
\hline ASA Class & \multicolumn{2}{|c|}{$N=457$} & \multicolumn{2}{|c|}{$N=323$} & \multicolumn{2}{|c|}{$N=48$} & \multicolumn{2}{|c|}{$N=86$} & \\
\hline $\mathrm{I}$ & 373 & 81.6 & 259 & 80.2 & 35 & 72.9 & 79 & 91.9 & 0.035 \\
\hline II & 72 & 15.8 & 54 & 16.7 & 12 & 25.0 & 6 & 7.0 & \\
\hline III, IV, V & 12 & 2.6 & 10 & 3.1 & 1 & 2.1 & 1 & 1.1 & \\
\hline \multicolumn{10}{|l|}{ Type of surgical procedure ${ }^{a}$} \\
\hline Abscess incision and drainage & 41 & 6.3 & 19 & 4.4 & 22 & 24.2 & 0 & 0 & Unable to report \\
\hline Closed reduction of fracture & 27 & 4.2 & 1 & 0.2 & 12 & 13.2 & 14 & 11.5 & \\
\hline Laparotomy & 31 & 4.8 & 27 & 6.2 & 3 & 3.3 & 1 & 0.8 & \\
\hline Excision of cysts, lipoma, keloid and mass & 169 & 26.0 & 152 & 34.9 & 9 & 9.9 & 8 & 6.6 & \\
\hline Wound care/skin graft & 47 & 7.2 & 26 & 6.0 & 16 & 17.6 & 5 & 4.1 & \\
\hline Hernia/hydrocele repair & 155 & 23.9 & 72 & 16.5 & 7 & 7.8 & 76 & 62.3 & \\
\hline Biopsy & 39 & 6.0 & 36 & 8.3 & 3 & 3.3 & 0 & 0 & \\
\hline Amputation & 18 & 2.8 & 11 & 2.5 & 4 & 4.4 & 3 & 2.5 & \\
\hline Hemorrhoidectomy & 26 & 4.0 & 21 & 4.8 & 1 & 1.1 & 4 & 3.3 & \\
\hline Mastectomy & 29 & 4.5 & 27 & 6.2 & 2 & 2.2 & 0 & 0 & \\
\hline Other & 67 & 10.3 & 44 & 10.1 & 12 & 13.2 & 11 & 9.0 & \\
\hline \multicolumn{10}{|l|}{ Received antibiotics } \\
\hline & $N=$ & & $N=$ & & $N=$ & & $N=$ & & \\
\hline Pre-operative & 123 & 25.6 & 60 & 17.8 & 49 & 72.1 & 14 & 18.7 & $<0.001$ \\
\hline & $N=$ & & $N=$ & & $N=$ & & $N=$ & & \\
\hline Peri-operative & 345 & 74.5 & 267 & 79.2 & 16 & 30.2 & 62 & 84.9 & $<0.001$ \\
\hline & $N=$ & & $N=$ & & $N=$ & & $N=$ & & \\
\hline Post-operative & 338 & 68.6 & 208 & 61.7 & 63 & 90.0 & 67 & 77.9 & $<0.001$ \\
\hline Type of anesthesia & $N=$ & & $N=$ & & $N=$ & & $N=$ & & \\
\hline Local & 118 & 20.2 & 111 & 26.6 & 5 & 7.1 & 2 & 2.1 & $<0.001$ \\
\hline Regional & 176 & 30.1 & 121 & 28.9 & 12 & 17.2 & 43 & 44.3 & \\
\hline General & 285 & 48.7 & 181 & 43.3 & 52 & 74.3 & 52 & 53.6 & \\
\hline Sedation Only & 5 & 0.8 & 4 & 1.0 & 1 & 1.4 & 0 & 0 & \\
\hline Other & 1 & 0.2 & 1 & 0.2 & 0 & 0 & 0 & 0 & \\
\hline Surgical provider ${ }^{\mathrm{a}}$ & $N=$ & & $N=$ & & $N=$ & & $N=$ & & \\
\hline General practitioner on staff & 231 & 36.6 & 33 & 7.6 & 80 & 98.8 & 118 & 100.0 & \\
\hline General surgeon on staff & 184 & 29.2 & 184 & 42.6 & 0 & 0 & 0 & 0 & \\
\hline Visiting general practitioner & 5 & 0.8 & 5 & 1.2 & 0 & 0 & 0 & 0 & \\
\hline Visiting general surgeon & 8 & 1.3 & 8 & 1.9 & 0 & 0 & 0 & 0 & \\
\hline Visiting plastic surgeon & 182 & 28.8 & 182 & 42.1 & 0 & 0 & 0 & 0 & \\
\hline Visiting orthopedic surgeon & 2 & 0.3 & 2 & 0.5 & 0 & 0 & 0 & 0 & \\
\hline Visiting obstetrician & 4 & 0.6 & 3 & 0.7 & 1 & 1.2 & 0 & 0 & \\
\hline Visiting breast surgeon & 15 & 2.4 & 15 & 3.5 & 0 & 0 & 0 & 0 & \\
\hline Duration of surgical procedure & $N=$ & & $N=$ & & $N=$ & & $N=$ & & \\
\hline
\end{tabular}


Table 2 continued

\begin{tabular}{|c|c|c|c|c|c|c|c|c|c|}
\hline & \multicolumn{2}{|c|}{ All the hospitals } & \multicolumn{2}{|c|}{ Butaro } & \multicolumn{2}{|c|}{ Rwinkwavu } & \multicolumn{2}{|c|}{ Kirehe } & \multirow[t]{2}{*}{$p$ value } \\
\hline & $n$ & $\%$ & $n$ & $\%$ & $n$ & $\%$ & $n$ & $\%$ & \\
\hline$\leq 30 \mathrm{~min}$ & 204 & 51.4 & 143 & 47.4 & 15 & 51.7 & 46 & 69.7 & $<0.001$ \\
\hline$>30$ and $\leq 60 \mathrm{~min}$ & 102 & 25.7 & 75 & 24.8 & 9 & 31.0 & 18 & 27.3 & \\
\hline$>1$ and $\leq 2 \mathrm{~h}$ & 58 & 14.6 & 52 & 17.2 & 4 & 13.8 & 2 & 3.0 & \\
\hline$>2 \mathrm{~h}$ & 33 & 8.3 & 32 & 10.6 & 1 & 3.5 & 0 & 0 & \\
\hline \multicolumn{10}{|l|}{ Surgical complications } \\
\hline Surgical site infection & 16 & 2.5 & 14 & 3.2 & 1 & 1.1 & 1 & 0.8 & 0.131 \\
\hline Unplanned reoperations & 3 & 0.5 & 3 & 0.7 & 0 & 0 & 0 & 0 & \\
\hline Other infections & 30 & 6.0 & 33 & 7.6 & 3 & 3.3 & 3 & 2.5 & \\
\hline None recorded & 591 & 91.0 & 386 & 88.5 & 87 & 95.6 & 118 & 96.7 & \\
\hline
\end{tabular}

min minutes; $h$ hours

${ }^{\text {a }}$ Stata could not calculate a Fisher's exact $p$ value

$(52.1 \%, n=513)$. The most common imaging overall was orthopedic related x-ray $(26.8 \%, n=713)$. The major non-surgical treatments were the use of both narcotic and non-narcotic pain medication $(73.8 \%, n=1964)$, antibiotics $(63.5 \%, n=1689)$, and wound care $(52.7 \%$, $n=1401)$, and these differed significantly across the hospitals $(p<0.001)$. The majority of the patients were discharged between 1 and 7 days $(n=1468,58.2 \%)$.

\section{Outcomes}

Overall, we found that $562(21.1 \%)$ patients were operated upon and were discharged, and the additional $562(21.1 \%)$ were referred to another facility (Table 4). Up to 1051 $(39.5 \%)$ patients received non-operative management only and were discharged, $39(1.5 \%)$ absconded, and 34 $(1.3 \%)$ died. Outcomes were not recorded for 412 $(15.5 \%)$ patients. Cancer patients $(n=103,40.9 \%)$ and non-cancer general surgery patients $(n=299,58.8 \%)$ were most likely to receive surgery and be discharged. Most trauma patients $(n=572,55.9 \%)$ and those with surgical infections $(n=312,57.8 \%)$ were discharged with non-operative management, and the majority of the patients with congenital conditions $(n=19,59.4 \%)$ were referred to another facility.

\section{Discussion}

In this study, we retrospectively investigated medical records of patients needing non-obstetric surgical care in three rural district hospitals in Rwanda. Most patients were males, and injury was the most common diagnosis, mainly resulting from road traffic injuries and assaults. Injury has been recognized as the leading condition requiring surgical care in Rwanda and other LMICs $[1,2,8]$. The prevalence in our cohort is similar to a Kenyan study where $41 \%$ of patients who visited a hospital's emergency department were injured [9]. The Kenyan study also found that most non-obstetric surgical patients were males and most injuries resulted from road traffic injuries $(42 \%)$ and assaults (28\%), consistent with our results. Road traffic injuries are a persistent menace in Africa, and preventive strategies at the national level such as improved road traffic safety and community promotion of the safe use of roads are suggested [1].

Supporting laboratory and imaging diagnostics were limited, with only $59.5 \%$ of patients having any laboratory testing and only $45.0 \%$ receiving any imaging. While not all patients presenting for surgical care may need testing or imaging (for example, in Kenya, only $72 \%$ of trauma patients needed x-rays [9], the current level of testing and diagnostics remains low. As observed in other studies, inadequate equipment and materials may contribute to the low levels of laboratory tests and imaging [2]. In addition, the variation noted in diagnostics may be attributed to differences in equipment and availability of specialists at the three district hospitals. Availing the needed equipment and materials can improve laboratory testing and imaging and hence improve diagnosis for better care. These differences may also reflect variation in practice patterns based on the surgical training received by the providers at the different district hospitals. Developing protocols for management of surgical conditions to guide providers at all district hospitals could also improve appropriate resource utilization for surgical patients.

For patients seeking non-obstetric surgical care at the district hospitals, nearly a quarter received some type of operation at the district hospital. Although other studies report over $80 \%$ of major surgeries happening at the district hospitals in Rwanda (CHUK Report 2010), most of these are obstetric cases [6, 7]. Although obstetrics care 
Table 3 Non-operative management-evaluation and treatment

\begin{tabular}{|c|c|c|c|c|c|c|c|c|c|}
\hline & \multicolumn{2}{|c|}{$\begin{array}{l}\text { All the hospitals } \\
N=2660\end{array}$} & \multicolumn{2}{|c|}{$\begin{array}{l}\text { Butaro } \\
N=766\end{array}$} & \multicolumn{2}{|c|}{$\begin{array}{l}\text { Rwinkwavu } \\
N=910\end{array}$} & \multicolumn{2}{|c|}{$\begin{array}{l}\text { Kirehe } \\
N=984\end{array}$} & \multirow[t]{2}{*}{$p$ value } \\
\hline & $n$ & $\%$ & $n$ & $\%$ & $n$ & $\%$ & $n$ & $\%$ & \\
\hline Received at least one laboratory test ${ }^{\mathrm{a}}$ & 1583 & 59.5 & 649 & 84.7 & 437 & 48.2 & 497 & 50.5 & $<0.001$ \\
\hline Full blood count & 1447 & 54.4 & 618 & 80.7 & 394 & 43.3 & 435 & 44.2 & $<0.001$ \\
\hline Bleeding time & 474 & 17.8 & 360 & 47.0 & 33 & 3.6 & 81 & 8.2 & $<0.001$ \\
\hline Clotting time & 457 & 17.2 & 356 & 46.5 & 37 & 4.1 & 64 & 6.5 & $<0.001$ \\
\hline HIV & 913 & 34.3 & 527 & 68.8 & 190 & 20.9 & 196 & 19.9 & $<0.001$ \\
\hline Type and cross & 527 & 19.8 & 377 & 49.2 & 68 & 7.5 & 82 & 8.3 & $<0.001$ \\
\hline At least one imaging test ${ }^{a}$ & 1197 & 45.0 & 266 & 34.7 & 418 & 45.9 & 513 & 52.1 & $<0.001$ \\
\hline Abdomen x-ray & 77 & 2.9 & 35 & 4.6 & 17 & 1.9 & 25 & 2.5 & 0.004 \\
\hline Orthopedic $\mathrm{x}$-ray ${ }^{\mathrm{b}}$ & 713 & 26.8 & 168 & 21.9 & 238 & 26.2 & 308 & 31.3 & $<0.001$ \\
\hline Chest x-ray & 247 & 9.3 & 67 & 8.8 & 105 & 11.5 & 75 & 7.6 & 0.012 \\
\hline Head x-ray & 249 & 9.4 & 30 & 3.9 & 90 & 9.9 & 129 & 13.1 & $<0.001$ \\
\hline Ultrasound & 166 & 6.2 & 47 & 6.1 & 87 & 9.6 & 32 & 3.3 & $<0.001$ \\
\hline \multicolumn{10}{|l|}{ Non-operative treatments ${ }^{\mathrm{a}}$} \\
\hline Antibiotics & 1689 & 63.5 & 560 & 73.2 & 532 & 60.5 & 597 & 63.2 & $<0.001$ \\
\hline Non-OR wound care & 1401 & 52.7 & 371 & $48 . .5$ & 419 & 46.1 & 611 & 62.1 & $<0.001$ \\
\hline Pain medication (narcotic + non-narcotic) & 1964 & 73.8 & 638 & 83.3 & 546 & 60.0 & 780 & 79.3 & $<0.001$ \\
\hline Traction for fracture & 25 & 0.9 & 21 & 2.7 & 1 & 0.1 & 3 & 0.3 & $<0.001$ \\
\hline Casting/immobilization & 282 & 10.6 & 44 & 5.7 & 79 & 8.7 & 159 & 16.2 & $<0.001$ \\
\hline Blood transfusion & 52 & 1.9 & 18 & 2.4 & 22 & 2.4 & 12 & 1.2 & 0.095 \\
\hline Admission ward of patient & \multicolumn{2}{|c|}{$N=2639$} & \multicolumn{2}{|c|}{$N=762$} & \multicolumn{2}{|c|}{$N=895$} & \multicolumn{2}{|c|}{$N=973$} & \\
\hline Surgery ward & 2163 & 82.0 & 685 & 89.9 & 605 & 67.6 & 873 & 89.7 & $<0.001$ \\
\hline Other wards & 467 & 18.0 & 77 & 10.1 & 290 & 32.4 & 100 & 10.3 & \\
\hline Duration in the hospital $^{c}$ & \multicolumn{2}{|c|}{$N=2525$} & \multicolumn{2}{|c|}{$N=752$} & \multicolumn{2}{|c|}{$N=856$} & \multicolumn{2}{|c|}{$N=917$} & \\
\hline Discharged same day & 314 & 12.4 & 33 & 4.4 & 221 & 25.8 & 60 & 6.5 & Unable to report \\
\hline 1-7 days & 1468 & 58.2 & 444 & 59.0 & 433 & 50.6 & 591 & 64.5 & \\
\hline 8-14 days & 328 & 13.0 & 122 & 16.2 & 93 & 10.9 & 113 & 12.3 & \\
\hline 15-30 days & 245 & 9.7 & 99 & 13.2 & 58 & 6.8 & 88 & 9.6 & \\
\hline More than 30 days & 170 & 6.7 & 54 & 7.2 & 51 & 6.0 & 65 & 7.1 & \\
\hline
\end{tabular}

We defined non-operative management as treatment, such as laboratory tests, imaging, narcotic and non-narcotic pain medication, wound care, and the use of antibiotics, that did not involve surgical operation

${ }^{a}$ Each test and treatment is a binary variable and only reporting the frequencies for those who received an intervention

b Includes extremity, and pelvic and spine x-rays

c Stata could not calculate a Fisher's exact $p$ value

may affect non-obstetrics care at the district hospital level [10], due to separate obstetrics theaters and providers in our study hospitals, we suspect minimal impact and advise further study to assess this relationship. There was significant variation in types of non-obstetric surgery completed across the three district hospitals, and similar variations at district hospitals have been observed in other sub-Saharan countries [6]. Kirehe and Rwinkwavu DH had higher incidences of orthopedic trauma (56 and $49 \%$ of all nonobstetric conditions, respectively) compared to Butaro $(18 \%)$. This pattern remained even with the exclusion of operations performed by visiting plastic surgeons which might skew the surgical presentations (Kirehe $(56 \%)$, Rwinkwavu (49\%), and Butaro (23\%)). Butaro is more rural and difficult to reach than Kirehe and Rwinkwavu which are closer to tarmac roads, and hence higher road traffic and more likely to experience road traffic injuries. The differing surgical practice patterns at district hospitals reflect differences in both surgical capacity and epidemiologic needs at each hospital, indicating that decision makers will need to tailor some decisions based on the needs at the hospital level.

A significantly higher proportion of patients in Butaro DH received surgery, likely attributable to the presence of 
Table 4 Immediate administrative outcomes of patients needing non-obstetric surgical care

\begin{tabular}{|c|c|c|c|c|c|c|c|c|c|c|c|c|c|c|c|c|}
\hline & \multirow{2}{*}{\multicolumn{2}{|c|}{$\begin{array}{l}\text { All surgical } \\
\text { patients }\end{array}$}} & \multicolumn{14}{|c|}{ Diagnosis } \\
\hline & & & \multicolumn{2}{|c|}{ Trauma } & \multicolumn{2}{|c|}{ Infection } & \multicolumn{2}{|c|}{ Cancer } & \multicolumn{2}{|c|}{$\begin{array}{l}\text { General } \\
\text { surgery }\end{array}$} & \multicolumn{2}{|c|}{ Congenital } & \multicolumn{2}{|c|}{ Urology } & \multicolumn{2}{|c|}{ Unknown } \\
\hline & $n$ & $\%$ & $n$ & $\%$ & $n$ & $\%$ & $n$ & $\%$ & $n$ & $\%$ & $n$ & $\%$ & $n$ & $\%$ & $n$ & $\%$ \\
\hline \multicolumn{17}{|l|}{ Outcomes } \\
\hline Received operation and discharged & 562 & 21.1 & 41 & 4.0 & 67 & 12.4 & 103 & 40.9 & 299 & 58.8 & 7 & 21.9 & 11 & 22.5 & 34 & 13.3 \\
\hline $\begin{array}{l}\text { Received non-operative management } \\
\text { only and discharged }\end{array}$ & 1051 & 39.5 & 572 & 55.9 & 312 & 57.8 & 35 & 13.9 & 40 & 7.9 & 2 & 6.3 & 14 & 28.6 & 76 & 29.8 \\
\hline Referred to another facility & 562 & 21.1 & 206 & 20.1 & 80 & 14.8 & 78 & 31.0 & 90 & 17.7 & 19 & 59.4 & 8 & 16.3 & 81 & 31.8 \\
\hline Absconded & 39 & 1.5 & 29 & 2.8 & 4 & 0.7 & 1 & 0.4 & 2 & 0.4 & 0 & 0 & 1 & 2.0 & 2 & 0.8 \\
\hline Died & 34 & 1.3 & 7 & 0.7 & 10 & 1.9 & 6 & 2.4 & 1 & 0.2 & 0 & 0 & 2 & 4.1 & 8 & 3.1 \\
\hline Unknown & 412 & 15.5 & 169 & 16.5 & 67 & 12.4 & 29 & 11.5 & 76 & 15.0 & 4 & 12.5 & 13 & 26.5 & 54 & 21.2 \\
\hline
\end{tabular}

a general surgeon on staff in Butaro in addition to frequent specialist surgeons' visits who provided non-routine surgical care in this setting during 2013. In most countries in sub-Saharan Africa, hospitals rely on non-surgeons to provide surgical care in rural facilities. A study of eight district hospitals in Mozambique, Tanzania, and Uganda found no specialist surgeons or anesthetists, with general practitioners and nurses providing most of the operative services [4]. While not all patients presenting with a surgical condition need an operative procedure, the very low volume of operative procedures, especially in Rwinkwavu and Kirehe, indicates a gap in meeting the district-level surgical need. The increased number of operations at Butaro DH emphasizes recommendations that more operative care could be provided at the district hospital level if additional surgical staff were available and proper surgical equipment availed [1]. In Butaro, visiting plastic surgeons performed $40 \%$ of all surgeries in 2013. However, the exclusion of the 182 surgeries performed by the visiting surgeons still left Butaro with the highest volume of operations, more than twice the operations performed in Rwinkwavu or Kirehe, suggesting that the visits met local needs rather than skew presentation and interventions. The 2015 Lancet Commission on Global Surgery also recommends surgical task sharing through increasing the number of non-physician surgeons as a temporary solution [1]. Not only would this reduce the number of patients discharged without receiving a needed procedure, but it would also reduce the number of patients referred to tertiary facilities, which are heavily burdened by the number of surgical referrals. However, there is need to ensuring that the additional trained surgical staff are comfortable with handling the most common surgical needs. Although trauma was the major clinical diagnosis overall, the majority of the trauma cases were referred. Currently, orthopedic care in Rwanda is available at referral hospitals. A general surgeon, without additional training and equipment, would not be able to handle the majority of orthopedic cases, depending on complexity.

Several limitations should be considered in the interpretation of these results. As our study used routinely collected data from patients' charts, we were not able to report for all variables on all patients. We performed data quality checks to reduce inaccuracies; however, missing data were often due to data not being recorded in the clinical charts. We believe that these data are missing at random, due to the heavy clinical load at the hospitals. However, we recommend revising the clinical charts and implementing routine audits to improve these data in the future. Finally, even though our study was limited to three rural district hospitals and that Butaro DH was relatively different from Rwinkwavu and Kirehe DHs due to the presence of a surgeon on staff and other visiting surgical specialists, the general infrastructure and human resources available at Rwinkwavu and Kirehe DHs are similar to other district hospitals in Rwanda. Therefore, we believe our results are informative of the needs of other rural district hospitals in Rwanda and beyond.

\section{Conclusion}

We found variation in the operative and non-operative surgical care that was provided at each district hospital potentially reflecting the variation in the surgical capacity of each district hospital in terms of human resources as well as equipment. As shown by our results, the percentage of patients receiving operative care was higher in Butaro than in Rwinkwavu and Kirehe. We attribute this to the presence of a general surgeon on staff and more surgical equipment. In the absence of trained surgeons particularly in low-resource settings, surgical task sharing is an 
alternative option [1, 11]. However, robust comparative analysis, assessing the relationship between the presence of surgeons and surgical equipment with surgical volume, interventions, and outcomes, is recommended.

Rwanda's Ministry of Health is implementing a Human Resources for Health program to increase the number of specialist throughout the country, including surgical providers, in addition to improving rural district hospital's infrastructure $[12,13]$. While these initiatives should go far in improving surgical care in the rural districts over time, assessing local needs and providing training to meet those needs could address the site-specific issues immediately at hand. Countries responding to the Lancet Commission call to create national surgical plans can learn from the care provided and the existing gaps identified in this study to appropriately address their surgical needs.

Acknowledgments We acknowledge Partners In Health/Inshuti Mu Buzima and the IMB Innovation Grants for the support of this work. This study was developed under the Partners In Health/Inshuti Mu Buzima Intermediate Operational Research Training Program, developed and facilitated by Bethany Hedt-Gauthier and Neil Gupta. We are thankful to all three PIH/IMB-supported District Hospitals staff for their contribution. We also appreciate the Association of Academic Surgeons and the Harvard Kennedy Traveling Fellowship for their financial support of the data collection for this project.

Author contributions $\mathrm{EM}$ and $\mathrm{CH}$ led the study design, data cleaning and analysis, results interpretation, and manuscript preparation. RR, BHG, JO, RM, and NG provided inputs in study design, data analysis, and results interpretation and critically reviewed the manuscript. BHG, RM, and JO supported data cleaning, and RR, BHG, and NG also provided mentorship. GT, TN, TM, and JN supported study design, results interpretation, and manuscript preparation.

\section{Compliance with ethical standards}

Conflict of interest The authors affirm that they have no competing interests.

\section{References}

1. Meara JG, Leather AJM, Hagander L et al (2015) Global surgery 2030: evidence and solutions for achieving health, welfare, and economic development. The Lancet 386(9993):569-624

2. Grimes CE, Bowman KG, Dodgion CM et al (2011) Systematic review of barriers to surgical care in low-income and middleincome countries. World J Surg 35:941-950

3. Stewart B, Khanduri P, McCord C et al (2014) Global disease burden of conditions requiring emergency surgery. Br J Surg 101:e9-e22

4. Kruk ME, Wladis A, Mbembati N et al (2010) Human resource and funding constraints for essential surgery in district hospitals in Africa: a retrospective cross-sectional survey. PLoS Med 7(3): 1000242

5. Hsia RY, Mbembati NA, Macfarlane S et al (2011) Access to emergency and surgical care in sub-Saharan Africa: the infrastructure gap. Health Policy Plan 27:234-244

6. Galukande M, Von Schreeb J, Wladis A et al (2010) Essential surgery at the district hospital: a retrospective descriptive analysis in three African countries. PLoS Med 7(3):e1000243

7. Petroze RT, Nzayisenga A, Rusanganwa V et al (2012) Comprehensive national analysis of emergency and essential surgical capacity in Rwanda. Br J Surg 99:436-443

8. Linden AF, Maine RG, Hedt-Gauthier BL et al (2016) Validation of a community-based survey assessing nonobstetric surgical conditions in Burera District, Rwanda. Surgery 159(4):1217-1226

9. Ogendi JOK, Ayisi JG (2011) Causes of injuries resulting in a visit to the emergency department of a Provincial General Hospital, Nyanza, western Kenya. Afr Health Sci 11(2):255-261

10. Petroze RT, Mehtsun W, Nzayisenga A, Ntakiyiruta G, Sawyer RG, Calland JF (2010) Ratio of cesarean sections to total procedures as a marker of district hospital trauma capacity. World J Surg 36(9):2074-2079

11. Chu K, Rosseel P, Gielis P et al (2009) Surgical task shifting in sub-Saharan Africa. PLoS Med 6(5):e1000078

12. Binagwaho A, Kyamanywa P, Farmer PE et al (2013) The human resources for health program in rwanda-a new partnership. N Engl J Med 369(21):2054-2059

13. Higashi H, Barendregt JJ, Kassebaum NJ et al (2014) Burden of injuries avertable by a basic surgical package in in low-and middle-income regions: a systematic analysis from the Global Burden of Disease 2010 study. World J Surg 39:1-9 